\title{
Association between frailty status and cognitive performance of elderly patients attending the Family Medicine Outpatient Clinic in Ismailia, Egypt
}

\author{
RABAB ATTA SAUDI ${ }^{A-E}$, EMAN ESMAT TOSSON ${ }^{A, B, E, F}$, HEND MIKHAIL SALAMA $^{\mathrm{A}, \mathrm{D}, \mathrm{E}, \mathrm{F}}$ \\ ORCID ID: 0000-0001-5477-9476 \\ ORCID ID: 0000-0001-6968-2538
}

Family Medicine Department, Faculty of Medicine, Suez Canal University, Ismailia City, Egypt

A - Study Design, B - Data Collection, C - Statistical Analysis, D - Data Interpretation, E - Manuscript Preparation, F - Literature

Search, G - Funds Collection

Summary Background. Frailty and cognitive impairment are serious problems affecting older adults. Screening for frailty in primary health care decreases unplanned secondary care use. There is still controversy about the association of frailty with cognitive impairment.

Objectives. This study aimed to assess the prevalence of frailty among aging patients, to evaluate the association between physical frailty and cognitive function among elderly patients who presented to the Family Medicine Outpatient Clinic and to determine the most impaired cognitive domains among frail patients.

Material and methods. This is a descriptive-analytical cross-sectional study. It included 404 elderly patients attending the Family Medicine Outpatient Clinic in Ismailia, Egypt. Study participants were classified into three frailty groups according to Fried criteria. The Montreal cognitive function (MoCA) and Mini-Mental State Examination (MMSE) tools were used to assess cognitive function. A multinomial logistic regression analysis was performed to investigate the association between performance in the cognitive (MoCA) domains and frailty.

Results. $19.3 \%$ of the study participants were frail. Frailty was associated with cognitive impairment by both MMSE and MoCA tests. About $85.69 \%$ of frail elderly patients had cognitive impairment. Frailty showed a significant association with lower global MoCA scores $(\mathrm{OR}=0.54, p<0.05)$. A specific MoCA cognitive domain: poor attention ( $O R=0.47, p<0.05)$, was a positive predictor of frailty. Conclusions. Frailty has been associated with lower total scores of both MMSE and MoCA. Specific impaired MoCA cognitive domains [fluency, orientation, calculation, abstraction, delayed recall, visuo-perception, naming and attention] were significantly associated with frailty status.

Key words: frailty, cognitive dysfunction, aged, mental status and dementia tests.

Saudi RA, Tosson EE, Salama HM. Association between frailty status and cognitive performance of elderly patients attending the Family Medicine Outpatient Clinic in Ismailia, Egypt. Fam Med Prim Care Rev 2021; 23(2): 232-238, doi: https://doi.org/10.5114/ fmpcr.2021.105933.

\section{Background}

Aging is associated with lowered cognitive and physical function. Frailty is defined as medical syndromes identified by physical decline and diminished age-related physiologic reserve leading to a reduction in the resistance to stressors and an increase in the risk of morbidity, disability and mortality [1]. Others define frailty as a person who cannot cope with stress, leading to adverse outcomes like falls, hospitalizations, disability and mortality [2].

Despite the fact that the definition of frailty is not yet clear [1], the most common method to measure frailty is the Fried frailty phenotype. Fried suggested 5 objective components of the criteria, including unintentional weight loss, muscle weakness, exhaustion, slowness while walking and inactivity, and at least 3 criteria of these 5 components are needed to identify frailty [2].

However, there is no consensus about the best definition and criteria to identify the syndrome and whether cognitive impairment should be added to frailty criteria [3]. It was proposed that the existing definition of frailty that focuses on physical functioning should be extended to include other aspects of health, such as cognitive function [4]. However, frailty is a common geriatric condition, its prevalence differs depending on how frailty is identified, which ranges from $4 \%$ up to $59 \%$ in community-dwelling older adults [5].

Frailty is a significant indicator of health and well-being and might be a superior indicator of negative outcomes in older adults [6]. A study was conducted to assess the risk factors of disability in the last year of life and reported that frailty was the most frequent disorder leading to death [7]. Cognitive impairment $(\mathrm{Cl})$ is defined as a decreased intellectual function that ranges widely from forgetfulness to dementia. Although mild cognitive impairment $(\mathrm{MCl})$ is a mild decline in cognitive function, which is not associated with significant functional disability, it increases the possibility of suffering from dementia, in which there is severe cognitive decline accompanied by decreased physical function, which ultimately leads the patients to be completely physically dependent on others [8]. By 2050, about 115 million people worldwide are expected to have dementia. $\mathrm{Cl}$ is associated with a raised risk of slow walking speed and future frailty [9]. Though many studies are now focusing on the frailty- $\mathrm{Cl}$ relationship, their literature had generally considered them as two different entities. Given the many risk factors and underlying mechanisms common to physical frailty and $\mathrm{Cl}$ [10], the International Academy on Nutrition and Aging and the International Association of Gerontology and Geriatrics had proposed a new term, "cognitive frailty" (CF) - a clinical diagnosis 
that explains the simultaneous presence of physical frailty and $\mathrm{MCl}[3]$.

Although there is no universal consensus regarding $\mathrm{CF}$, it has been used in many recent studies, where it has been associated with a greater risk of adverse outcomes [10]. Frailty may increase the risk of mild cognitive impairment $(\mathrm{MCl})$ and dementia [11]. Furthermore, elements of frailty have seemed to be associated with pathological findings of vascular and Alzheimer's disease (AD), supporting the concept of a possible combined biological pathway between frailty and cognitive disorders [12].

The main feature in the Egyptian population over the last decades is a gradual increase in life expectancy for both males and females. Egypt has the highest percentage of elderly in the Arab world (7.2\%), and this proportion is expected to increase to $9.9 \%$ in 2030 and $15.3 \%$ in 2050 [13]. Such a rapid increase in the elderly population would pose a variety of challenges, such as inadequate health care, inadequate pension, poverty, polypharmacy and inadequate health insurance coverage. The elderly in Egypt finance most of their healthcare consumption through out-of-pocket expenses [14]. Identification of frailty in primary health care is important, because many interventions, such as high-intensity exercise training and nutritional supplementation, can improve the degree of frailty and thus decrease the risk of morbidity, disability and mortality. With the rapidly aging population, primary care physicians will be challenged to recognize and manage frail elders and their associated chronic conditions with judicious use of the limited available geriatric specialist resources. Research on frailty assessment and its association with poor cognition in Egypt is scarce, especially among the aging population attending a Family Medicine setting.

\section{Objectives}

This study aimed to assess the prevalence of frailty of aging patients, to evaluate the association between physical frailty and cognitive function among elderly patients who presented to the Family Medicine Outpatient Clinic and to determine the most impaired cognitive domains among frail patients.

\section{Material and methods}

\section{Study design and data collection}

The current study is an analytical cross-sectional study including a descriptive component. This study was carried out in the Family Medicine Outpatient Clinic affiliated with Suez Canal University Hospitals in the Ismailia Governorate. The study was conducted from January 2018 to June 2019.

\section{Participants}

The study was conducted on elderly patients 60 years of age or older who met the inclusion criteria and who attended the Family Medicine Outpatient Clinic during the study period.

\section{Inclusion criteria}

Elderly patients of both genders, 60 years of age or more, who agreed to participate in the study and were able to answer the questionnaires during the interview were included.

\section{Exclusion criteria}

The study excluded patients known to have any underlying condition, such as Parkinson's disease or stroke or diagnosed severe depression that made the patient unsuitable for the study.

\section{Sample size}

It was planned to take a sample sufficient enough to demonstrate a $39.6 \%$ prevalence of cognitive impairment among both pre-frail and frail elderly adults [15]. To ensure that the $95 \%$ confidence interval estimate of the proportion of the sample is within $5 \%$ of the true proportion, by calculation, the sample size was equal to $367+10 \%$ drop out, with a total sample of 404 elderly patients.

\section{Study tools and questionnaires}

The study tools consisted of a Comprehensive Geriatric Assessment in the form of structured interview questionnaires, which were previously translated and validated in Arabic language, and included the following items:

A) Demographic characteristics and health status including age, gender, marital status, education, occupation, living condition (living alone or not) and smoking (current smoker or not). Clinical characteristics included general health status (self-reported), history of falls during the past 6 months, self-reported medical history of having ever been diagnosed with chronic diseases (hypertension, diabetes mellitus, osteoporosis, cardiac diseases, chronic obstructive pulmonary disease, kidney disease cancer, thyroid disease, stroke, Parkinson's disease, eye disorders and joint disorders (osteoarthritis and rheumatoid arthritis)) and polypharmacy (defined as taking four or more medications, determined by asking about the current number of prescribing medicine).

B) Frailty assessment: Fried frailty criteria were used to assess the frailty status according to the frailty phenotype, which was derived from the Cardiovascular Health Study [2]. The 5-component version includes weight loss, exhaustion, slowness, weakness and inactivity.

- Weight loss: defined as an unintentional weight loss of $>10$ lbs. ( $\geq 4.5 \mathrm{~kg}$ ) or loss $\geq$ of $5 \%$ of body mass in the last year.

- Exhaustion: the following two questions were posed using the scale of the Center for Epidemiological Studies Depression (CES-D) [16]: 1. How many days did you feel that all you've done was a major effort in the last week?; 2. How many times did you think you couldn't keep on doing things in the last week? Answers were scored between 0 and 4 [ $0=$ rarely or no time $(<1$ day), 1 = some time ( $1-2$ days), 2 = average time (3-4 days), and $3=$ most of the time]. Answers of " 2 " or " 3 " were considered as positive.

- $\quad$ Slowness: was defined by the 3-meter walking speed test (interpretation of results takes into account gender and height).

Males:

Height $<173 \mathrm{~cm} \ldots<0.76 \mathrm{~m} / \mathrm{s}$ Height $>173$ $\mathrm{cm} \ldots<0.65 \mathrm{~m} / \mathrm{s}$

Females:

Height $<159 \mathrm{~cm} \ldots<0.76 \mathrm{~m} / \mathrm{s}$ Height $>159$ $\mathrm{cm} . . .<0.65 \mathrm{~m} / \mathrm{s}$

If gait speed is lower than these respective cut-offs, the criterion is positive.

- Inactivity: was defined as walking for $<3 \mathrm{~h} /$ week over the past 12 months.

- Weakness assessed by a self-response as "yes" to "Do you feel weaker in the last 12 months or think your strength has diminished?"

Categorization of frailty according to Fried criteria: Patients who fulfilled none of these criteria were considered non-frail, patients who fulfilled one or two criteria were classified as pre-frail, and patients who fulfilled three or more criteria were classified as frail. 


\section{C) Assessment of cognitive function:}

1. Mini-Mental State Examination (MMSE) is the most common tool used worldwide for cognitive impairment and dementia screening. The MMSE is a quick and easy measure that assesses seven areas of cognitive functioning (orientation, memory "immediate and short-term", attention and calculation, language and praxis). The Arabic version of MMSE was shown to be valid and reliable, with a sensitivity and specificity of $60.9 \%$ and $59.5 \%$, respectively [17]. The cutoff point for abnormal MMSE was < 24 , and the results were correlated with the age and educational level of the participants.

2. Montreal Cognitive Assessment (MoCA) was developed as a screening tool for mild cognitive impairment $(\mathrm{MCl})$. MoCA examines the domains of executive functions, language, orientation, calculations, memory, visuo-constructional skills, attention and concentration and concept. al thinking. The total score is 30 points. A score more than or equal to 26 was considered normal, and for lower educated individuals, 1 point was added to the total MoCA score for those with 12 years or less of education. The Arabic MoCA tool revealed good internal consistency (Cronbach's alpha $=0.915$ ) and content validity in segregation between normal and diseased subjects. When screening for mild cognitive impairment among aging persons, the MoCA test has displayed a greater sensitivity and specificity compared to the MMSE (MoCA-B $=0.988$ versus MMSE = 0.939) [18]. MoCA was designed as screening instrument for mild cognitive impairment, mild cognitive impairment, which could be missed by MMSE due to higher sensitivity and specificity.

D) Geriatric depression scale: 15 items used for screening for depression among aging persons. The Arabic GDS-15 form has good psychometric features, but the best properties were decelerated for the $7 / 8$ cutoff. The Arabic version of the GDS-15 has displayed a good internal consistency (Cronbach's alpha $=0.88$ ) [19].

E) Functional assessment: activities of daily living (ADL) (personal care, clothing, moving, going to the toilet, eating) were measured with the Katz scale, and the total score ranges from 0 to 6 , with higher scores meaning better function [20]. Lawton's assessment scale was used to assess abilities in instrumental activities of daily living (IADL), such as making phone calls, shopping, driving and using money). The total score ranges from 0 to 8 , with higher score meaning better physical function [21].

\section{Ethical considerations}

All procedures performed in the study were in accordance with the ethical standards of the institutional research committee and with the 1964 Helsinki declaration and its later amendments. Administrative permissions were granted by the center's manager, who was informed about the aim of the study. The participants were assured that their refusal to participate or withdraw at any time did not affect the integrity of their care in the primary healthcare center. Informed written consent was signed by every patient after clarifying the aim of the study. The confidentiality of data was assured.

\section{Data analysis}

The Statistical Package for Social Sciences (SPSS) version 20 was used for data analysis. The Shapiro-Wilk test was used to test continuous data for normality. Descriptive characteristics were outlined as means, standard deviations (SD) or median, interquartile range (IQR) for continuous variables (where appropriate) and percentages for categorical variables. The participants in the study were divided into three frailty classes (non-frail, pre-frail and frail). Pearson's chi-square test was used to compare independent categorical data. The analyses of variance (ANOVA) test or Kruskal-Wallis test was used to detect discrepancies between frailty groups, where appropriate. Multinomial logistic regression was used to assess the association between frailty status and impaired cognitive performance. We considered the non-frail group as a reference in the regression models. Multiple linear regression models were used to detect how changes in frailty components (independent variables) affected the total MoCA score (dependent variable). All requested variables were entered (frailty, weight loss, slow gait speed, weakness, exhaustion and inactivity). The $p$-value was statistically significant if $\leq 0.05$ and $<0.017$ for multiple comparisons.

\section{Results}

Table 1 demonstrates that our study included 404 elderly patients with a mean age of $66.5 \pm 4.9$. Nearly half of the study sample was female $(215(53.2 \%))$, the majority of them had a low educational level $(325(80.4 \%))$, and $19.3 \%$ of the study sample was classified as frail, while $34.7 \%$ as pre-frail. There were statistically significant relationships between frailty and being older, male, having lower education, not being married, having a lower income, having more comorbidities and polypharmacy. Furthermore, frailty was associated with poor general health status, history of falls and a lower body mass index. Frailty was also significantly related to lower scores in both activities of daily living and instrumental activities of daily living. Moreover, higher depression scores were related to frailty.

\begin{tabular}{|c|c|c|c|c|c|}
\hline Variables & $\begin{array}{l}\text { Total } \\
404(100)\end{array}$ & \begin{tabular}{|l} 
Non-frail 186 \\
(46)
\end{tabular} & $\begin{array}{l}\text { Pre-frail } 140 \\
(34.7)\end{array}$ & \begin{tabular}{|l|} 
Frail \\
78 (19.3)
\end{tabular} & $p$ \\
\hline Age (years): mean (SD) & $66.5(4.9)$ & $62.9(2.7)$ & $68.4(3.9)$ & $71.6(4.2)$ & $<0.001^{\mathrm{a}}$ \\
\hline Gender (female): $n(\%)$ & $215(53.2)$ & $91(48.9)$ & $88(62.9)$ & $36(46.2)$ & $0.02^{\mathrm{b}}$ \\
\hline Address (urban): $n(\%)$ & $223(55.2)$ & $101(54.3)$ & $79(56.4)$ & $43(55.2)$ & $0.92^{\mathrm{b}}$ \\
\hline Education years ( $\leq 6$ years): $n(\%)$ & $325(80.4)$ & $136(73.1)$ & $118(84.3)$ & $71(17.6)$ & $<0.001^{b}$ \\
\hline Marital status (single/widow/divorced): $n$ (\%) & $167(41.3)$ & $51(27.4)$ & $67(47.9)$ & $49(62.8)$ & $<0.001^{b}$ \\
\hline Insufficient income: $n(\%)$ & $261(64.6)$ & $113(60.8)$ & $88(62.9)$ & 60 (76.9) & $0.03^{b}$ \\
\hline Current smoking: $n(\%)$ & $98(24.3)$ & $45(24.2)$ & $28(20)$ & $25(32.1)$ & $0.14^{b}$ \\
\hline Lives alone: $n(\%)$ & $34(8.4)$ & $15(8.1)$ & $13(9.3)$ & $6(7.7)$ & $0.8^{\mathrm{b}}$ \\
\hline $\begin{array}{l}\text { Number of chronic illness: } \\
\text { median (IQR) }\end{array}$ & $2(1-3)$ & $2(1-2)$ & $2(2-3)$ & $3(2-4)$ & $<0.001^{\mathrm{c}}$ \\
\hline Polypharmacy ( $\geq 4$ drugs): $n$ (\%) & $175(43.3)$ & $51(27.4)$ & $71(50.7)$ & $53(67.9)$ & $<0.001^{b}$ \\
\hline
\end{tabular}




\begin{tabular}{|c|c|c|c|c|c|c|}
\hline Variables & $n(\%)$ & \begin{tabular}{|l} 
Total \\
$404(100)$
\end{tabular} & \begin{tabular}{|l}
$\begin{array}{l}\text { Non-frail } 186 \\
(46)\end{array}$ \\
\end{tabular} & $\begin{array}{l}\text { Pre-frail } 140 \\
(34.7)\end{array}$ & $\begin{array}{l}\text { Frail } \\
78(19.3)\end{array}$ & $p$ \\
\hline Poor general health status: $n(\%)$ & & $106(26.2)$ & $8(4.3)$ & $46(32.9)$ & $52(66.7)$ & $<0.001^{\mathrm{b}}$ \\
\hline History of falls in past year: $n(\%)$ & & $126(31.2)$ & $44(23.7)$ & $57(40.7)$ & $25(32.1)$ & $0.004^{b}$ \\
\hline BMI: mean (SD) & & $24.5(4.6)$ & $26.4(3.7)$ & $24.1(4.4)$ & $20.9(4.3)$ & $<0.001^{\mathrm{b}}$ \\
\hline ADL score: mean (SD) & & $4.9(1.2)$ & $5.8(0.4)$ & $4.7(0.8)$ & $3.1(1.1)$ & $<0.001^{\text {a }}$ \\
\hline IADL score: median (IQR) & & $4(2-6)$ & $6(5-7)$ & $3(2-5)$ & $2(0-3)$ & $<0.001^{\circ}$ \\
\hline Depression (GDS): median (IQR) & & $3(2-5)$ & $3(2-4)$ & $3(3-7)$ & $5(2-7)$ & $<0.001^{\circ}$ \\
\hline
\end{tabular}

${ }^{a}$ One-way ANOVA test, ${ }^{b}$ Chi-square test, ${ }^{c}$ Kruskall-Wallis test.

Statistical significance at $p<0.05$ and $p<0.017$ for multiple comparisons.

BMI - Body Mass Index, ADL - Activities of Daily Living, IADL - Instrumental Activities of Daily Living, GDS - Geriatric Depression Score.

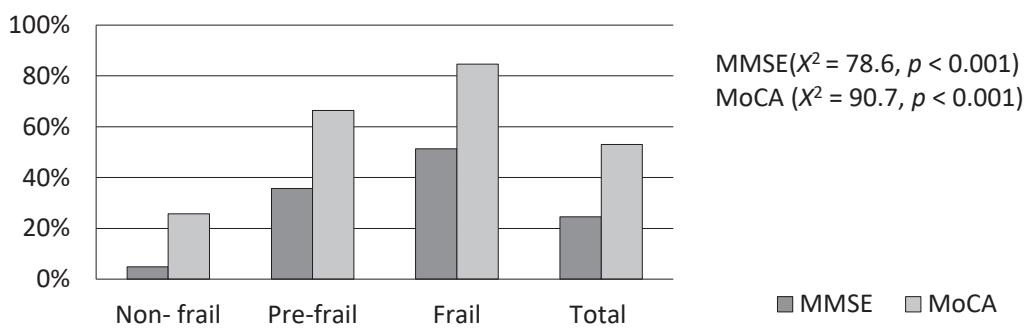

Figure 1. Cognitive impairment among frailty groups

Figure 1 shows that about one-quarter of the sample (24.5\%) had cognitive impairment measured by MMSE, which increased to (52.5\%) when measured by MoCA. Frail and pre-frail elderly patients had a higher prevalence of cognitive impairment in both MMSE $(51.3 \%, 35.7 \%, p<0.001)$ and MoCA $(85.69 \%, 66.4 \%, p<0.001)$ compared to the non-frail group.
Table 2 demonstrates that there is significant association between frailty and lower total scores of both MMSE and MoCA ( $p<$ 0.001). Furthermore, impaired specific MoCA cognitive domains (fluency, orientation, calculation, abstraction, delayed recall, visuo-perception, naming and attention $(p<0.001)$ and abstraction $(p<0.05))$ were significantly associated with frailty status.

\begin{tabular}{|l|l|l|l|l|l|l|}
\hline \multicolumn{7}{|l}{ Table 2. Association between physical frailty and cognitive functions in the study sample $(\boldsymbol{n}=404)$} \\
\hline $\begin{array}{l}\text { Variables: } \\
\text { Median (IQR) }\end{array}$ & Total & Non-frail & Pre-frail & Frail & $\begin{array}{l}\text { Kruskal- Wallis } \\
\text { Test }\left(X^{2}\right)\end{array}$ & $p$ \\
\hline MMSE score & $26(24-28)$ & $28(27-30)$ & $25(22-27)$ & $22(18-26)$ & 142.1 & $<0.001$ \\
\hline MoCA score & $24(20-27)$ & $26(24-30)$ & $20(16.5-26)$ & $18(14-22)$ & 109.1 & $<0.001$ \\
\hline Executive function & $1(0-1)$ & $1(1-1)$ & $1(1-1)$ & $1(1-1)$ & 3.1 & 0.22 \\
\hline Fluency & $2(1-2)$ & $2(2-2)$ & $2(1-2)$ & $1(0-2)$ & 80.5 & $<0.001$ \\
\hline Orientation & $6(4-6)$ & $6(6-6)$ & $5(4-6)$ & $4(3-6)$ & 92.9 & $<0.001$ \\
\hline Calculation & $2(1-3)$ & $2(1-3)$ & $2(1-2)$ & $1.5(1-2)$ & 39.4 & $<0.001$ \\
\hline Abstraction & $3(2-3)$ & $3(3-3)$ & $3(2-3)$ & $2.5(2-3)$ & 10.2 & 0.01 \\
\hline Delayed recall & $3(2-4)$ & $4(3-6)$ & $3(2-4)$ & $3(2-3)$ & 65.8 & $<0.001$ \\
\hline Visuo-perception & $2(1-3)$ & $3(2-3)$ & $2(1-3)$ & $1(1-2)$ & 71.2 & $<0.001$ \\
\hline Naming & $4(3-4)$ & $4(4-4)$ & $3(2-4)$ & $3(2-4)$ & 84.2 & $<0.001$ \\
\hline Attention & $2(1-3)$ & $3(2-3)$ & $2(1-3)$ & $1(1-2)$ & 93.9 & $<0.001$ \\
\hline
\end{tabular}

MMSE - Mini-Mental State Examination, MoCA - Montreal Cognitive Assessment, Statistical significance at $p<0.05$ and $p<0.017$ for multiple comparisons.

\begin{tabular}{|c|c|c|c|c|c|c|c|c|}
\hline \multirow[t]{3}{*}{ Variable } & \multicolumn{8}{|l|}{ Groups } \\
\hline & \multicolumn{4}{|c|}{ Pre-Frail } & \multicolumn{4}{|l|}{ Frail } \\
\hline & B & SE & Wald & OR $(95 \% \mathrm{CI})$ & B & SE & Wald & OR $(95 \% \mathrm{CI})$ \\
\hline MoCA total & -0.140 & 0.199 & 0.498 & 0.869 (0.589-1.28) & -0.617 & 0.251 & 6.05 & $0.540(0.330-0.882)^{*}$ \\
\hline Executive function & -0.286 & 0.450 & 0.404 & $0.751(0.311-1.81)$ & -0.289 & 0.591 & 0.239 & $0.749(0.235-2.38)$ \\
\hline Fluency & -0.583 & 0.378 & 2.39 & $0.558(0.266-1.17)$ & 0.312 & 0.473 & 0.437 & $1.36(0.541-3.45)$ \\
\hline Orientation & -0.123 & 0.283 & 0.189 & $0.884(0.508-1.54)$ & 0.091 & 0.332 & 0.075 & $1.09(0.572-2.09)$ \\
\hline Calculation & 0.242 & 0.278 & 0.756 & $1.27(0.739-2.19)$ & 0.688 & 0.351 & 3.84 & $1.99(1.00-3.95)$ \\
\hline Abstraction & 0.085 & 0.279 & 0.093 & $1.09(0.630-1.88)$ & 0.425 & 0.376 & 1.28 & $1.52(0.732-3.19)$ \\
\hline
\end{tabular}




\begin{tabular}{|c|c|c|c|c|c|c|c|c|}
\hline \multirow[t]{3}{*}{ Variable } & \multicolumn{8}{|c|}{ Groups } \\
\hline & \multicolumn{4}{|c|}{ Pre-Frail } & \multicolumn{4}{|l|}{ Frail } \\
\hline & B & SE & Wald & OR $(95 \% \mathrm{Cl})$ & B & SE & Wald & OR $(95 \% \mathrm{Cl})$ \\
\hline Delayed recall & 0.239 & 0.261 & 0.839 & $1.26(0.762-2.12)$ & 0.538 & 0.332 & 2.63 & $1.71(0.894-3.28)$ \\
\hline Visuo-perception & 0.268 & 0.304 & 0.778 & $1.31(0.721-2.37)$ & 0.132 & 0.372 & 0.125 & $1.14(0.550-2.36)$ \\
\hline Naming & -0.446 & 0.290 & 2.37 & $0.640(0.362-1.13)$ & 0.373 & 0.362 & 1.06 & $1.45(0.714-2.95)$ \\
\hline Attention & -0.619 & 0.295 & 4.40 & $0.538(0.302-0.960)^{*}$ & -0.753 & 0.357 & 4.44 & $0.47(0.234-0.949)^{*}$ \\
\hline
\end{tabular}

The reference category is: non-frail group; (Likelihood ratio $X^{2}=213.8$; * $p<0.001$; Pseudo $R^{2}=0.25$ ).

$* p<0.05$, MoCA - Montreal Cognitive Assessment.

\begin{tabular}{|c|c|c|c|c|c|c|}
\hline \multirow[t]{2}{*}{ Frailty components } & \multicolumn{2}{|l|}{ Model 1} & \multicolumn{2}{|l|}{ Model 2} & \multicolumn{2}{|l|}{ Model 3} \\
\hline & $\begin{array}{l}\text { B-coefficient } \\
(95 \% \text {-CI) }\end{array}$ & $p$ & $\begin{array}{l}\text { 6-coefficient } \\
(95 \%-\mathrm{CI})\end{array}$ & $p$ & $\begin{array}{l}\text { B-coefficient } \\
(95 \%-\mathrm{CI})\end{array}$ & $p$ \\
\hline Frailty & $-0.45(-0.651--0.41)$ & $<0.001^{*}$ & $-0.45(-0.649--0.41)$ & $<0.001^{*}$ & $-0.44(-0.642--0.41)$ & $<0.001^{*}$ \\
\hline Weight loss & $-0.11(-4.02-0.58)$ & $0.007^{*}$ & $-0.07(-3.09-0.129)$ & $0.012^{*}$ & $-0.076(-3.23-0.160)$ & $0.01^{*}$ \\
\hline Exhaustion & $-0.12(-3.25-0.49)$ & $0.024 *$ & $-0.070(-2.55-0.903)$ & $0.023^{*}$ & $-0.068(-2.52-0.940)$ & $0.023 *$ \\
\hline Slow gait speed & $-0.06(-2.43-0.92)$ & 0.371 & $0.010(-1.43-1.69)$ & 0.871 & $0.010(-1.435-1.69)$ & 0.873 \\
\hline Inactivity & $-0.09(-2.91-0.83)$ & 0.270 & $-0.009(-1.85-1.64)$ & 0.903 & $-0.009(-1.84-1.64)$ & 0.910 \\
\hline Weakness & $-0.08(-3.17-0.75)$ & 0.222 & $-0.070(-2.88-0.746)$ & 0.242 & $-0.071(-2.89-0.743)$ & 0.246 \\
\hline
\end{tabular}

All requested variables entered. Dependent Variable: (MoCA). Values are presented as 6 -coefficient ( $95 \%$ confidence interval).

Model 1: frailty components variables (frailty, weight loss, exhaustion, slow gait, inactivity and weakness), $r$-square $=0.37, \mathrm{Model} A N O V A-F=39.44$, $p<0.001$.

Model 2: adjusted for age and gender, $r$-square $=0.47$, Model ANOVA: $F=44.49, p<0.001$.

Model 3: adjusted for comorbidities and general health status, $r$-square $=0.47$, Model ANOVA: $F=35.64 ; p<0.001$.

Table 3 illustrates the results of multinomial logistic regression. The frail group showed a significant association with lower global MoCA scores (OR $=0.540 ; 95 \% \mathrm{Cl}: 0.33-0.88 ; p<0.05)$. The specific cognitive MoCA domain that was statistically significantly associated with frailty status was poor attention (OR = 0.47; 95\% Cl: 0.234-0.949; $p<0.05$ ).

Table 4 shows the results of multiple linear regression models for the association of cognition (MoCA) scores with frailty components (frailty, weight loss, exhaustion, slow gait, inactivity and weakness) and revealed that after adjusting for age, gender, chronic diseases and self-reported general health status, frailty, weight loss and exhaustion were significantly associated with poor global cognition.

\section{Discussion}

In the current study, among 404 elderly patients who were included in the study, $46 \%$ of them were classified as non-frail, $34.7 \%$ as pre-frail and $19.3 \%$ as frail. The results of the current study were in agreement with several studies that had been conducted to evaluate the prevalence of frailty among aging populations. The high prevalence of frailty in our study was consistent with the results of a cross-sectional study that had been conducted in Egypt in 2015, which found that the prevalence of pre-frail and frail elders were $20.87 \%$ and $34.78 \%$, respectively [22]. Another study was conducted in Brazil to assess the prevalence of frailty and pre-frailty among the elderly population and found that the prevalence was $59.9 \%$ and $26.6 \%$, respectively [23]. The prevalence rate of cognitively pre-frail and cognitively frail among the multi-ethnic older population in Malaysia was $39.6 \%$ [15], while a nationwide survey in China found the standard overall prevalence of physical frailty, pre-frailty, cognitive impairment and cognitive frailty among the Chinese elderly population was $8.8,33.8,6.5$ and $2.0 \%$, respectively [6]. The high prevalence of frailty status in our study can be explained in light of the fact that our participants were outpatients, and it was found that frail patients may use health services more than those who are not frail.

In the current study, the frail group was more likely to be elderly males with a low educational level, while a study that was conducted by De Moris et al. showed that frail elderly persons were more likely to be elderly females with low schooling [23].

In the current study, the frailty group had the greatest number of chronic diseases and the highest polypharmacy levels, and more reported a poor general health status (all $p$-values were $<0.05$ ). This result is consistent with a study which reported that multiple subclinical and age-related comorbidities may worsen the decline in many physiological systems in older adults, causing homeostatic imbalance or frailty, brain aging and thus cognitive decline [24]. In the current study, it was found that frail and pre-frail elderly adults had a higher cognitive impairment percentage in both MMSE $(51.3 \%, 35.7 \%)$ and MoCA $(85.69 \%, 66.4 \%)$. This result was in agreement with the findings of several studies which reported that physical frailty is associated with the onset of cognitive impairment, consistently revealing a higher prevalence of cognitive impairment among frail patients $[9,24]$.

A cross-sectional study which was conducted by Amer et al. to evaluate the correlation between frailty status and cognition among community-dwelling non-demented elderly adults in Egypt found that those who were frail had a greater percentage of cognitive impairment, as $62.5 \%$ of the frail group had cognitive impairment in MMSE, while about $97.5 \%$ of the frail group had cognitive impairment according to MoCA [22]. A crosssectional analysis which was conducted by Yoon et al. reported that frail and pre-frail elderly individuals were more susceptible to progressive cognitive impairment, with about $90 \%$ of frail elderly individuals being cognitively impaired [25]. 
In our study, the performance of frail elderly individuals was bad considering global cognition. Furthermore, frailty was associated with lower scores in the domains of MoCA (fluency, orientation, calculation, abstraction, delayed recall, visuo-perception, naming and attention), and multinomial logistic regression also showed that frailty was associated with lower total MoCA score. Specific MoCA cognitive domain; poor attention was a positive predictor of frailty. These results were consistent with the findings of $\mathrm{Ma}$ et al., who conducted a study among communitydwelling elderly individuals and reported that frail participants had worse global cognition scores in all the domains of the MMSE than both non-frail and pre-frail individuals, and pre-frail residents had statistically lower scores in the areas of global cognition, reading, drawing, writing, repetition, orientation, recall and attention than non-frail elderly individuals [1]. On the other hand, Yoon et al. stated that frailty was associated with impaired cognitive function in particular domains of working memory, cognitive flexibility, processing speed and memory [25]. Another study that was conducted by Bunce et al., who assessed associations between specific cognitive domains and frailty syndrome, found that at baseline, a frail elderly persons showed poorer cognitive function in processing speed, word and face recognition, verbal fluency, episodic memory and reaction time [26]. Furthermore, the results of the study conducted by De Morais et al. on the association between frailty and poor global cognitive function showed impaired cognitive function particularly in cognitive domains such as thinking, memory and language [23].

This discrepancy in researches findings could be explained by the methodological variations to define frailty groups. Furthermore, the authors of these previous studies also prioritized other instruments to assess specific cognitive domains concerning frailty $[23,25,26]$.

In the current study, a multiple linear regression model showed that frailty, weight loss and exhaustion were significantly associated with global cognition, while slow gait speed and inactivity were insignificantly associated with cognition. These findings were compatible with the findings of the study conducted by Yoon et al., which reported that global cognition was significantly lower in individuals with frailty, exhaustion, slowness and inactivity [25]. In the Irish Longitudinal Study on Ageing, it was reported that slow gait as an individual component of the frailty phenotype was correlated with impaired cognitive function, as well as, prospectively, non-Alzheimer disease in an elderly frail person [8]. In Korea, the results of a study which was conducted among elderly females suggested that cognition was associated with factors linked to frailty syndrome, such as age, handgrip strength, low walking speed and depression [27]. These results could be explained by the recently, published neuropathology data which have shown additional links between cognitive impairment and frailty, suggesting common mechanisms. Another study stated that cognitive impairment and physical frailty were associated with an increased fear of falls [28].
One of the limitations of this study was that we could not establish causality in the association between physical frailty and cognition, as this is a cross-sectional study. However, based on findings from previous studies that showed that physical frailty affects cognition, we suggest that physical frailty can be a risk factor for cognitive impairment. Another limitation of the study is the enrollment of outpatient elders, which may vary from the general elderly population. Thus, the frailty rate in this study may have been overestimated. Moreover, enrollment may signify the association of frailty and cognitive impairment. Third, some criteria of frailty were examined using self-reported data, which is subject to bias, such as weakness, which was examined by a question rather than measuring grip strength.

It is important for family physicians to incorporate the concept of frailty in the care of their older patients. Family medicine basically depends on a patient-centered approach that considers individual goals of care, beliefs, cultures, preferences, social context and previous experiences of the patient with illness. The introduction of the frailty concept would increase these core values and skills when caring for patients with frailty. In the context of complexity, feeling of concern and the challenges that often occur in caring for frail elderly individuals in overcrowded family physician's office, the physician can start making clinical decisions and recommendations about treatment in the light of frailty and its accompanying risks. This allows for targeted interventions and decreases inappropriate interventions. Moreover, family medicine is best suited to act on detecting those at risk for frailty as part of its preventive tasks [29].

\section{Conclusions}

In conclusion, comprehensive geriatric assessment is pivotal in the family medicine setting, as frailty is prevalent and is associated with a cognitive decline among elderly patients. This study provides epidemiological information about the prevalence of frailty and cognitive impairment among elderly Egyptians, which is prevalent in the family medicine setting. In addition, the study found an association between frailty and impaired global cognitive function. Further impaired specific MoCA cognitive domains (fluency, orientation, calculation, abstraction, delayed recall, visuo-perception, naming and attention $(p<0.001)$ and abstraction $(p<0.05))$ were significantly associated with frailty status. Aging community-dwellers should be monitored closely for frailty and cognitive decline. Despite the fact that causal associations between frailty and cognitive decline are currently unclear, longitudinal studies are needed to determine the causal relationship between frailty in aging individuals and cognitive impairment.

Acknowledgements. The authors would like to acknowledge all the participants for their co-operation.

Source of funding: This work was funded from the authors' own resources.

Conflicts of interest: The authors declare no conflicts of interest.

\section{References}

1. Ma L, Zhang L, Tang Z, et al. Use of the frailty index in evaluating the prognosis of older people in Beijing: a cohort study with an 8-year follow-up. Arch Gerontol Geriatr 2016; 64: 172-177.

2. Fried LP, Tangen CM, Walston J, et al. Frailty in older adults: evidence for a phenotype. J Gerontol A Biol Sci 2001; 56: M146-M156.

3. Kelaiditi $\mathrm{E}$, Cesari M, Canevelli M, et al. Cognitive frailty: rational and definition from an (I.A.N.A./I. A.G.G.) international consensus group. J Nutr Heal Aging 2013; 17: 726-734, doi: 10.1007/s12603-013-0367-2.

4. Langlois F, Vu Thien M, Kergoat M, et al. The multiple dimensions of frailty: physical capacity, cognition, and quality of life. Int Psychogeriatr 2012; 24: 1429.

5. Collard R, Boter H, Schoevers R, et al. Prevalence of frailty in community-dwelling older persons: a systematic review. J Am Geriatr Soc 2012; 60: 1487-1492.

6. Ma L. Current situation of frailty screening tools for older adults. J Nutr Heal Aging 2018; 1: 8, doi: 10.1007/s12603-018-1123-4.

7. Gill TM, Gahbauer EA, Han L, et al. Trajectories of disability in the last year of life. N Engl J Med 2010; 362: 1173-1180, doi: 10.1056/ NEJMoa0909087. 
8. Robertson DA, Savva GM, Kenny RA. Frailty and cognitive impairment: a review of the evidence and causal mechanisms. Ageing Res Rev 2013; 12: 840-851.

9. Feng L, Zin Nyunt MS, Gao Q, et al. Cognitive frailty and adverse health outcomes: findings from the Singapore longitudinal aging studies (SLAs). J Am Med Dir Assoc 2017; 18: 252-258, doi: 10.1016/j. jamda.2016.09.015.

10. Fougère B, Delrieu J, Del Campo N, et al. Cognitive frailty. Mechanisms, tools to measure, prevention, and controversy. Clin Geriatr Med 2017; 33: 339-355, doi: 10.1016/j.cger.2017.03.001.

11. Boyle PA, Buchman AS, Wilson RS, et al. Physical frailty is associated with incident mild cognitive impairment in community-based older persons. J Am Geriatr Soc 2010; 58: 248-255, doi: 10.1111/j.1532 5415.2009.02671.x.

12. Buchman AS, Yu Lei, Wilson RS, et al. Brain pathology contributes to simultaneous change in physical frailty and cognition in old age. J Gerontol A Biol Sci 2014; 69: 1536-1544, doi: 10.1093/gerona/glu117.

13. World Population Ageing Report. United Nations, New York, 2015. Department of Economic and Social Affairs. Population Division 2015. Available at URL: http://www.UN.com.

14. Maurizio B, Koettl J, Emily S. Golden aging: prospects for healthy, active, and prosperous aging in Europe and Central Asia. World Bank Group; 2015. Available from URL: http://hdl.handle.net/10986/22018.

15. Rivan MNF, Shahar S, Rajab NF, et al. Cognitive frailty among Malaysian older adults: baseline findings from the LRGS TUA cohort study. Clin Interv Aging 2019; 14: 1343-1352, doi: 10.2147/CIA.S211027.

16. Orme JG, Reis J, Herz EJ. Factorial and discriminant validity of the Center for Epidemiological Studies Depression (CES-D) scale. J Clin Psychol 1986; 42: 28-33

17. Albanna M, Yehya A, Khairi A, et al. Validation and cultural adaptation of the Arabic versions of the Mini-Mental Status Examination-2 and Mini-Cog test. Neuropsychiatr Dis Treat 2017; 14: 793-801.

18. Saleh AA, Alkholy RSAEHA, Khalaf OO, et al. Validation of montreal cognitive assessment-basic in a sample of elderly Egyptians with neurocognitive disorders. Aging Ment Health 2019; 23: 551-557, doi: 10.1080/13607863.2018.1428936.

19. Chaaya M, Sibai A, Roueiheb Z, et al. Validation of the Arabic version of the short Geriatric Depression Scale (GDS-15). Int Psychogeriatr 2008; 20(3): 571-581, doi: 10.1017/S1041610208006741.

20. Katz S, Ford AB, Moskowitz RW, et al. Studies of illness in the aged. The index of ADL: a standardized measure of biological and psychosocial function. JAMA 1963; 21(9): 914-919.

21. Lawton MP, Brody EM. Assessment of older people: self-maintaining and instrumental activities of daily living. Gerontologist 1969; 9(3): 179-186.

22. Amer MS, El Akkad RM, Hassan HS. Association of cognitive impairment with frailty in the elderly: a cross-sectional study on community-dwelling non-demented elderly referred to an outpatient geriatric service in Egypt. Indian J Appl Res 2015; 5(9): $210-215$.

23. De Morais FD, Da Silva AT, Nishihara CMH. Frailty and cognitive performance in older adults living in the community: a cross-sectional study. Arch Clin 2019; 46(6): 151-155, doi: 10.1590/0101-60830000000216.

24. Clegg A, Young J, lliff S, et al. Frailty in elderly people. Lancet 2013; 381(9868): 752-762, doi: 10.1016/ S0140-6736(12)62167-9.

25. Yoon DH, Hwang SS, Lee DW, et al. Physical frailty and cognitive functioning in Korea rural community-dwelling older adults. $J$ Clin Med 2018; 7: 405.

26. Bunce D, Batterham PJ, Mackinnon AJ. Long-term associations between physical frailty and performance in specific cognitive domains. J Gerontol B Psychol Sci Soc Sci 2019; 74(6): 919-926.

27. Kang JY, Kim CH, Sung EJ, et al. The association between frailty and cognition in elderly women. Korean J Fam Med 2016; 37(3): 164-170, doi: 10.4082/kjfm.2016.37.3.164.

28. Kurková $\mathrm{P}$, Kisvetrová $\mathrm{H}$, Horváthová $\mathrm{M}$, et al. Fear of falling and physical performance among older Czech adults. Fam Med Prim Care Rev 2020; 22(1): 32-35, doi: 10.5114/fmpcr.2020.92503.

29. Monteserin R, Brotons C, Moral I, et al. Effectiveness of a geriatric intervention in primary care: a randomized clinical trial. Fam Pract 2010; 27: 239-245, doi: 10.1093/fampra/cmp101.

Tables: 4

Figures: 1

References: 29

Received: 29.04 .2020

Reviewed: 4.05 .2020

Accepted: 6.07.2020

Address for correspondence:

Rabab Atta Saudi, MD

Department of Family Medicine

Faculty of Medicine

Suez Canal University

Ismailia

Egypt

Tel.: +20 1156222864

E-mail: rabab.atta82@gmail.com 